\title{
AN INTEGRAL TRANSFORM SOLUTION OF THE DIFFERENTIAL EQUATION FOR THE TRANSVERSE MOTION OF AN ELASTIC BEAM
}

\author{
by J. FULTON \\ (Received 22nd December 1956)
}

\section{Introduction}

It is well known* that certain types of partial differential equation may be solved using integral transforms with suitable kernels. In general, these equations may be solved by the classical method of separating variables, but the use of an integral transform yields the solution in a more direct way in the sense that the boundary values are contained in the solution.

It is the purpose of this note to apply this technique to obtain the solution of the differential equation associated with the transverse motion of an elastic beam for a wide class of boundary conditions.

The inversion theorem for a finite integral transform is in the form of a series expansion of the characteristic functions of the differential system, and the normalisation of these functions may involve laborious integrations. It is shown in $\S 3$ how the expansion coefficients may be obtained algebraically, thereby simplifying the process.

\section{The Differential Equation} form

The partial differential equation with which we are concerned has the

$$
\frac{\partial^{2}}{\partial x^{2}}\left(p \frac{\partial^{2} y}{\partial x^{2}}\right)=P(x, t)-\rho \frac{\partial^{2} y}{\partial t^{2}}
$$

where $p(x)>0$, and $\rho(x)>0$ are continuous functions in the range $0 \leqslant x \leqslant l$.

Associated with this equation is the characteristic equation

$$
\frac{d^{2}}{d x^{2}}\left(p \frac{d^{2} \phi}{d x^{2}}\right)-\xi^{4} \rho \phi=0
$$

It may readily be shown that this equation is self-adjoint. A solution $\phi(x, \xi)$ of (2) satisfying certain linear homogeneous relations between the values of $\phi, \phi^{\prime}, \phi^{\prime \prime}, \phi^{\prime \prime \prime}$, at $x=0$ and $x=l$, and involving certain parameters, is used to define the kernel of the integral transform which will reduce equation (1) to an ordinary differential equation in $t$, containing the values of $y, y^{\prime}, y^{\prime \prime}, y^{\prime \prime \prime}, \dagger$ at the points $x=0$ and $x=l$.

From the solution of this equation in $t$ is obtained the solution of (1) by applying the inversion theorem discussed in the next section.

\footnotetext{
* See, for example, Sneddon (4), and Tranter (7).

E.M.S. - F

$\dagger$ 'denotes differentiation with respect to $x$.
} 
The solutions of specific problems are obtained by assigning suitable values to the parameters.

\section{The Inversion Theorem}

We consider now the differential equation

$$
\begin{gathered}
\frac{d^{2}}{d x^{2}}\left(\boldsymbol{p} \frac{d^{2} \phi}{d x^{2}}\right)-\xi^{4} \rho \phi=0, \\
p(x)>0, \rho(x)>0,0 \leqslant x \leqslant l .
\end{gathered}
$$

and we subject $\phi$ to the linear homogeneous boundary conditions

$$
b_{i}(\phi) \equiv \phi_{0}^{(i-1)} \cos \alpha_{i}+\left(p \phi^{\prime \prime}\right)_{0}^{(2-i)} \sin a_{i}=\overline{0}, i=1,2
$$

where $\phi_{0}^{(n)}$ denotes the value of the $n$th derivative of $\phi$ at $x=0$, and the $\alpha_{i}$ are parameters. There are similar conditions associated with the point $x=l$ in terms of $\phi_{l}^{(n)}$ and the parameters $\beta_{i}$, and these for convenience will be denoted by $b_{i}(\phi)=0, i=3,4$.

If $\phi_{j}(x, \xi), j=1, \ldots, 4$ is a fundamental set of solutions of equation (2), and if we write $b_{i}\left(\phi_{j}\right)$ as $b_{i j}$, then the Green's function of the system is given by (Ince (3), p. 259).

$$
G(x, z, \xi)=N(x, z, \xi) / D(\xi)
$$

where $D(\xi)$ denotes the determinant $\left|b_{i j}\right|, i, j=1, \ldots, 4$, and

$$
N(x, z, \xi)=\left|\begin{array}{ccccc}
g(x, z, \xi) & \phi_{1}(x, \xi) & \phi_{2}(x, \xi) & \phi_{3}(x, \xi) & \phi_{4}(x, \xi) \\
b_{1}(g) & b_{11} & b_{12} & b_{13} & b_{14} \\
b_{2}(g) & b_{21} & b_{22} & b_{23} & b_{24} \\
b_{3}(g) & b_{31} & b_{32} & b_{33} & b_{34} \\
b_{4}(g) & b_{41} & b_{42} & b_{43} & b_{44}
\end{array}\right|
$$

In this determinant

$$
g(x, z, \xi)=-\sigma \sum_{k=1}^{4} \phi_{k}(x, \xi) \chi_{k}(z, \xi)
$$

where $\sigma=\frac{1}{2}$, if $x<z$, and $\sigma=-\frac{1}{2}$, if $x>z$, and

$$
\chi_{k}(z, \xi)=\frac{1}{p(z)} \frac{\partial}{\partial \phi_{k}^{\prime \prime \prime}} \log W(z) \text {. }
$$

$W(z)$ denotes the Wronskian of $\phi_{j}(z, \xi), j=1, \ldots, 4$, with respect to $z$. It can be shown (Darboux (2), p. 114) that since equation (2) is self-adjoint, the $\phi_{j}(z, \xi)$ may be chosen such that

$$
\chi_{k}(z, \xi)=\sum_{j=1}^{4} c_{k j} \phi_{j}(z, \xi)
$$

where $c_{k j}=\left|(-1)^{k+1} \delta_{k, 5-j}\right|, \delta_{m n}$ denoting the Kronecker $\delta$-function.

It can also be shown (Titchmarsh (6), p. 5 ; Ince (3), p. 258) that if $F(x)$ is an integrable function then

$$
F(x)=-\frac{\rho(x)}{2 \pi i} \oint \int_{0}^{l} G(x, z, \xi) F(z) d z \cdot d\left(\xi^{4}\right)
$$


where the contour of integration in the $\xi$-plane encloses all the poles of $G(x, z, \xi)$, that is, the zeros of $D(\xi) / 4 \xi^{3}=w(\xi)$, say. The zeros of $w(\xi)$ are the characteristic numbers of the system and form an infinite sequence of real numbers (Temple (5), p. 260). It will be assumed that the zeros are simple.

If $\xi_{n}$ is a characteristic number, then the cofactor of $g\left(x, z, \xi_{n}\right)$ in $N\left(x, z, \xi_{n}\right)$ is zero, and so $N\left(x, z, \xi_{n}\right)$ is a continuous function of $x$ and $z$. Expanding $N\left(x, z, \xi_{n}\right)$ by the elements of the first column, and making use of (6), (7), and (8) we obtain

$$
\begin{aligned}
N\left(x, z, \xi_{n}\right) & =\sum_{r=1}^{4} \sum_{s=1}^{4}(-1)^{r+s+1}\left\{\sum_{k=1}^{2}(-1)^{k+1} B_{k r} b_{k, 5-s}\right\} \phi_{r}\left(x, \xi_{n}\right) \phi_{s}\left(z, \xi_{n}\right) \\
& =\sum_{r=1}^{4} \sum_{s=1}^{4}(-1)^{r+s+1}\left\{\sum_{k=3}^{4}(-1)^{k} B_{k r} b_{k, 5-s}\right\} \phi_{r}\left(x, \xi_{n}\right) \phi_{s}\left(z, \xi_{n}\right) . .
\end{aligned}
$$

in which $B_{i j}$ denotes the minor of $b_{i j}$ in the determinant $D(\xi)$. We have used the fact that $\sum_{k=1}^{4} B_{k r} b_{k, 5-s}=0$; this is so because $D(\xi)=0$ in the case $r+s=5$, and because it is an expansion in terms of alien minors in the case $r+s \neq 5$.

Furthermore,

$$
\phi\left(x, \xi_{n}\right)=\left|\sum_{k=1}^{4}(-1)^{k+1} B_{i k} \phi_{k}\left(x, \xi_{n}\right)\right|, i=1, \ldots, 4,
$$

are characteristic functions of the differential system, and by virtue of its self-adjointness we can write

$$
\begin{aligned}
N\left(x, z, \xi_{n}\right) & =-\lambda\left(\xi_{n}\right) \phi\left(x, \xi_{n}\right) \phi\left(z, \xi_{n}\right) \\
& =-\lambda\left(\xi_{n}\right) \sum_{r=1}^{4} \sum_{s=1}^{4}(-1)^{r+s+1} B_{i r} B_{i s} \phi_{r}\left(x, \xi_{n}\right) \phi_{s}\left(z, \xi_{n}\right)
\end{aligned}
$$

Equating elements of the matrices of the bilinear forms (10) and (12) gives

$$
\begin{aligned}
\lambda\left(\xi_{n}\right) & =\sum_{k=1}^{\left.\sum_{(-1}^{2}-1\right)^{k+1} B_{k r} B_{k, 5-s}} / B_{i r} B_{i, s} \\
& =\sum_{k=3}^{4}(-1)^{k} B_{k r} B_{k, 5-s} / B_{i r} B_{i s}
\end{aligned}
$$

Putting $r=s=j$, and writing $\lambda\left(\xi_{n}\right)=\lambda_{n}$, we get

$$
\begin{aligned}
\lambda_{n} & =\sum_{k=1}^{2}(-1)^{k+1} B_{k j} B_{k, 5-j} /\left(B_{i j}\right)^{2} \\
& =\sum_{k=3}^{4}(-1)^{k} B_{k j} B_{k, 5-j} /\left(B_{i j}\right)^{2}
\end{aligned}
$$

in which $B_{i j} \neq 0$. At least one minor $B_{i j}$ of $D(\xi)$ must be non-zero if the system is singly-compatible.

Equation (9) now becomes

$$
F(x)=\sum_{n=1}^{\infty} \frac{\lambda_{n}}{w_{\xi}\left(\xi_{n}\right)} \rho(x) \phi\left(x, \xi_{n}\right) \int_{0}^{l} \phi\left(z, \xi_{n}\right) F(z) d z
$$


in which $w_{\xi}(\xi)$ denotes the derivative of $w(\xi)$ with respect to $\xi$. Writing $F(x)=\rho(x) f(x)$ and $\psi\left(x, \xi_{n}\right)=\left\{\frac{\lambda_{n}}{w_{\xi}\left(\xi_{n}\right)}\right\}^{\frac{1}{2}} \phi\left(x, \xi_{n}\right)$, this becomes

$$
f(x)=\sum_{n=1}^{\infty} \psi\left(x, \xi_{n}\right) \int_{0}^{l} \rho(z) \psi\left(z, \xi_{n}\right) f(z) d z
$$

It may readily be shown that

$$
\int_{0}^{l} \rho(x) \psi\left(x, \xi_{n}\right) \psi\left(x, \xi_{m}\right) d x=\delta_{m n},
$$

so that the $\{\rho(x)\}^{\ddagger} \psi\left(x, \xi_{n}\right)$ form a complete orthonormal set. The convergence of the expansion (14) is discussed by Birkhoff ((1), p. 389).

If $\xi_{n}$ is a repeated root of $w(\xi)=0$, formula (14a) must be suitably modified by calculating the residues of $G(x, z, \xi)$ at $\xi_{n}$ accordingly.

\section{The Integral Transform}

We now define the integral transform to be used in the solution of (1). Taking $\psi\left(x, \xi_{n}\right)$, as defined in the previous section, to be the kernel of the transform, we write

$$
f\left(\xi_{n}\right)=\int_{0}^{l} \rho(z) \psi\left(z, \xi_{n}\right) f(z) d z,
$$

then the inversion theorem is given by $(14 b)$ as

$$
f(x)=\sum_{n=1}^{\infty} \vec{f}\left(\xi_{n}\right) \psi\left(x, \xi_{n}\right)
$$

where $\xi_{1}, \xi_{2} \ldots$ are the roots of $w(\xi)=0$.

\section{The Solution of the Differential Equation}

If we multiply $\partial^{2} / \partial x^{2}\left(p \partial^{2} y / \partial x^{2}\right)$ by $\psi\left(x, \xi_{n}\right)$ and integrate by parts with respect to $x$ between the limits $x=0$ and $x=l$, we obtain

$$
\left[\left\{\psi \cdot\left(p y^{\prime \prime}\right)^{\prime}-\left(p \psi^{\prime \prime}\right)^{\prime} y\right\}-\left\{\psi^{\prime}\left(p y^{\prime \prime}\right)-\left(p \psi^{\prime \prime}\right) y^{\prime}\right\}\right]_{0}^{l}+\xi_{n}^{4} \int_{0}^{l} \rho \psi y d x
$$

Now $\psi\left(x, \xi_{n}\right)$ satisfies the boundary conditions (3) and so

$$
\begin{array}{lll}
\psi_{o}^{(i-1)}=\delta_{i} \sin \alpha_{i}, & \left(p \psi^{\prime \prime}\right)_{0}^{(2-i)}=\delta_{i} \cos \alpha_{i}, \quad i=1,2 \\
\psi_{l}^{(i-1)}=\mu_{i} \sin \beta_{i}, & \left(p \psi^{\prime \prime}\right)_{l}^{(2-i)}=-\mu_{i} \cos \beta_{i}, i=1,2
\end{array}
$$

where

$$
\begin{aligned}
\delta_{i}\left(\xi_{n}\right) & =\psi_{0}^{(i-1)} \sin a_{i}-\left(p \psi^{\prime \prime}\right)_{0}^{(2-i)} \cos a_{i} \\
\mu_{i}\left(\xi_{n}\right) & =\psi_{l}^{(i-1)} \sin \beta_{i}-\left(p \psi^{\prime \prime}\right)_{l}^{(2-i)} \cos \beta_{i}, i=1,2
\end{aligned}
$$

and (17) becomes

$$
B\left(\xi_{n}, t\right)+\xi_{n}^{4} \bar{y}\left(\xi_{n}, t\right)
$$

where

$$
\bar{y}\left(\xi_{n}, t\right)=\int_{0}^{l} \rho(x) \psi\left(x, \xi_{n}\right) y(x, t) d x
$$


and

$$
\begin{aligned}
B\left(\xi_{n}, t\right)=\sum_{i=1}^{2}(-1)^{i}\left[\delta_{i}\left(\xi_{n}\right)\left\{\left(p y^{\prime \prime}\right)_{0}^{(2-i)} \sin a_{i}+y_{0}^{(i-1)} \cos a_{i}\right\}\right. & \\
& \left.+\mu_{i}\left(\xi_{n}\right)\left\{\left(p y^{\prime \prime}\right)_{l}^{(2-i)} \sin \beta_{i}+y_{l}^{(i-1)} \cos \beta_{i}\right\}\right]
\end{aligned}
$$

Thus if we multiply equation (1) by $\psi\left(x, \xi_{n}\right)$ and integrate with respect to $x$ between $x=0$ and $x=l$, we obtain

$$
B\left(\xi_{n}, t\right)+\xi_{n}^{4} \bar{y}\left(\xi_{n}, t\right)=\bar{P}\left(\xi_{n}, t\right)-\frac{d^{2}}{d t^{2}} \bar{y}\left(\xi_{n}, t\right)
$$

where $P\left(\xi_{n}, t\right)$ is the transform of $P(x, t) / \rho(x)$.

The solution of this equation in $t$ is (Sneddon (4), p. 116)

$$
\begin{aligned}
\bar{y}\left(\xi_{n}, t\right)=\bar{y}\left(\xi_{n}, 0\right) \cos \xi_{n}^{2} t & +\frac{1}{\xi_{n}^{2}} \bar{y}_{t}\left(\xi_{n}, 0\right) \sin \xi_{n}^{2} t \\
& +\frac{1}{\xi_{n}^{2}} \int_{0}^{t}\left\{P\left(\xi_{n}, \tau\right)-B\left(\xi_{n}, \tau\right)\right\} \sin \xi_{n}^{2}(t-\tau) d \tau
\end{aligned}
$$

where

$$
\bar{y}\left(\xi_{n}, 0\right)=\int_{0}^{l} \rho(x) y(x, 0) \psi\left(x, \xi_{n}\right) d x
$$

and

$$
\bar{y}_{t}\left(\xi_{n}, 0\right)=\int_{0}^{l} \rho(x) y_{t}(x, 0) \psi\left(x, \xi_{n}\right) d x
$$

in which $y_{t}(x, 0)$ is the value of the derivative of $y$ with respect to $t$ at $t=0$.

The solution $y(x, t)$ follows by applying the inversion theorem, giving

$$
y(x, t)=\sum_{n=1}^{\infty} \bar{y}\left(\xi_{n}, t\right) \psi\left(x, \xi_{n}\right)
$$

\section{Applications}

(a) Beam of uniform cross-section

In the case of a beam of uniform cross-section, (1) becomes

$$
\frac{\partial^{4} y}{\partial x^{4}}+\frac{1 \partial^{2} y}{\mathrm{a}^{2} \partial t^{2}}=\frac{P(x, t)}{E I}, \mathrm{a}^{2}=E I / \rho
$$

where $E I$ and $\rho$ are constants, and the characteristic equation is

$$
\frac{d^{4} \phi}{d x^{4}}-\xi^{4} \phi=0
$$

The fundamental set of solutions of this equation satisfying (7) and (8) are $\phi_{1}=\left(2 \xi^{3}\right)^{-\frac{1}{t}} \sinh \xi x, \phi_{2}=\left(2 \xi^{3}\right)^{-1} \sin \xi x, \phi_{3}=\left(2 \xi^{3}\right)^{-\frac{1}{2}} \cos \xi x, \phi_{4}=\left(2 \xi^{3}\right)^{-\frac{1}{2}} \cosh \xi x$.

We consider in the first instance the problem of a uniform beam of length $l$, hinged at the ends, with bending moments $M_{0}(t)$ and $M_{l}(t)$ specified at $x=0$ and $x=l$ respectively. Thus the end conditions are

$$
\begin{aligned}
& y(0, t)=0, y^{\prime \prime}(0, t)=M_{0}(t) / E I, \text { at } x=0 ; \\
& y(l, t)=0, y^{\prime \prime}(0, t)=M_{1}(t) / E I, \text { at } x=l .
\end{aligned}
$$

E.M.S.-F 2 
In order to remove the unspecified end conditions in $(21)$, we chose

$$
a_{1}=\beta_{1}=0, a_{2}=\beta_{2}=\frac{1}{2} \pi \text {. }
$$

This yields $D(\xi)$ for this particular problem. From this we obtain $\omega(\xi)$ which reduces to $\frac{1}{\xi^{2}} \sinh \xi l \sin \xi l$ and so the characteristic numbers are the roots of the equation

$$
\frac{1}{\xi^{2}} \sinh \xi l \sin \xi l=0,
$$

that is $\xi_{n}=\frac{n \pi}{l}$, where $n=1,2,3, \ldots$

We note that in $D(\xi), B_{12} \neq 0$, and so taking $i=1, j=2$ in formulæ (11) and (13a) we obtain the orthonormal set of characteristic functions

$$
\psi\left(x, \xi_{n}\right)=\sqrt{\frac{\overline{2}}{l}} \sin \left(\frac{n \pi x}{l}\right) .
$$

Making the appropriate substitutions in (18) and (21) gives

$$
B(n, t)=\sqrt{\frac{2}{l}} \frac{n \pi}{E I l}\left\{M_{0}(t)+M_{l}(t) \cos n \pi\right\} .
$$

Equation (23) then becomes

$$
\begin{aligned}
\bar{y}(n, t)=\bar{y} \cos \left(\frac{a n^{2} \pi^{2} t}{l^{2}}\right)+ & \frac{l^{2} \bar{y} t}{a n^{2} \pi^{2}} \sin \left(\frac{a n^{2} \pi^{2} t}{l^{2}}\right) \\
& +\frac{l^{2} a}{E I n^{2} \pi^{2}} \int_{0}^{t}\{P(n, \tau)-B(n, \tau)\} \sin \left(\frac{a n^{2} \pi^{2}(t-\tau)}{l^{2}}\right) d \tau,
\end{aligned}
$$

which is in agreement with Sneddon ((4), p, 116) in case $M_{0}(t)=M_{l}(t)=0$.

As a further example of the uniform beam we shall take the case in which the bending moment $M(t)$ and shearing stress $S(t)$ are specified at the end $x=0$, and the beam is free at the other end. The end conditions are then

$$
\begin{array}{ll}
y^{\prime \prime}(0, t)=M(t) / E I, & y^{\prime \prime \prime}(0, t)=S(t) / E I, \text { at } x=0 ; \\
y^{\prime \prime}(l, t)=0, & y^{\prime \prime \prime}(l, t)=0, \text { at } x=l .
\end{array}
$$

The unspecified end conditions in (21) are removed by taking

$$
a_{1}=a_{2}=\beta_{1}=\beta_{2}=\frac{1}{2} \pi
$$

and the characteristic numbers are the roots of

$$
\xi(1-\cosh \xi l \cos \xi l)=0 .
$$

The normalised characteristic function corresponding to the characteristic number $\xi_{n}$ is

$$
\begin{aligned}
\psi\left(x, \xi_{n}\right)=\{l \mathbf{l}(C s-S c)\}^{-1}\{S s( & \left.\sinh \xi_{n} x+\sin \xi_{n} x\right) \\
& \left.-(C s-S c)\left(\cosh \xi_{n} x+\cos \xi_{n} x\right)\right\},
\end{aligned}
$$

where we have written $C=\cosh \xi_{n} l, c=\cos \xi_{n} l, S=\sinh \xi_{n} l, s=\sin \xi_{n} l$.

We find also that

$$
B\left(\xi_{n}, t\right)=2 l^{-1}\left\{S(t)+\xi_{n} S s(C s-S c)^{-1} M(t)\right\}
$$


For the characteristic number zero we find by expanding (25) and (26) in series of powers of $\xi_{n}$ and letting $\xi_{n} \rightarrow 0$, that

$$
\psi(x, 0)=l^{-\frac{1}{2}}(3 x / l-2), B(0, t)=2 l^{-1}\{S(t)+3 M(t) / 2\} .
$$

Substituting in (23) and using (24) yields the required solution.

\section{(b) Truncated cone with circular cross-section}

Taking the $x$-axis along the axis of the cone and writing $m=\left(d_{0}-d_{l}\right) / d_{0}$ where $d_{0}$ and $d_{l}$ are the diameters of the cross-sections of the ends $x=0$ and $x=l$, equation (1) becomes

$$
\frac{\partial^{2}}{\partial x^{2}}\left\{(1-m x)^{4} \frac{\partial^{2} y}{\partial x^{2}}\right\}+\frac{1}{a^{2}}(1-m x)^{2} \frac{\partial^{2} y}{\partial t^{2}}=\frac{P(x, t)}{E I_{0}}, a^{2}=E I_{0} / \rho_{0},
$$

where $E I_{0}$ and $\rho_{0}$ are constants. The characteristic equation is

$$
\frac{d^{2}}{d x^{2}}\left\{(1-m x)^{4} \frac{d^{2} \phi}{d x^{2}}\right\}-\xi^{4}(1-m x)^{2} \phi=0,
$$

of which the fundamental solutions are

$$
\phi_{1}=\left\{m^{\frac{3}{2}} \xi(1-m x)\right\}^{-1} I_{2}\{2 \xi \sqrt{ }(1-m x)\},
$$

with similar expressions for $\phi_{2}, \phi_{3}, \phi_{4}$ in terms of $Y_{2}, J_{2}, K_{2}$ respectively where $I, Y, J, K$ denote Bessel functions in the usual notation.

The subsequent calculations do not differ in principle from those of the previous example, but, because of the complexity of the expressions, are not given here. From the point of view of numerical calculation it is probably better to leave the expressions in determinantal form.

\section{REFERENCES}

(1) G. D. Birkhoff, Trans. Amer. Math. Soc., 9 (1908).

(2) G. Darboux, Leçons sur la Théorie Générale des Surfaces, vol. II (Paris, 1915).

(3) E. L. Ince, Ordinary Differential Equations (London, 1926).

(4) I. N. Sneddon, Fourier Transforms (New York, 1951).

(5) G. Temple, Proc. London Math. Soc., (2), 29, 1929.

(6) E. C. Titchmarsh, Eigenfunction Expansions (Oxford, 1946).

(7) C. J. Tranter, Integral Transforms (London, 1951).

Department of Technical Mathematios

The University of EDINbURgh 